\title{
Uncertain Candidates, Valence, and the Dynamics of Candidate Position- Taking
}

\author{
Michael Bruter \\ London School of Economics \\ M.Bruter@lse.ac.uk \\ Robert S. Erikson \\ Columbia University \\ rse14@columbia.edu \\ Aaron Strauss \\ Princeton University \\ abstraus@princeton.edu
}

September 2009

\section{Accepted for publication in Public Choice}

An earlier version of this paper was presented at the Conference on the Dynamics of Partisan Position Taking, Binghamton University, March 22-23, 2007.

\begin{abstract}
This paper offers graphic illustration of so-called "valence" models of candidates responding to their perceptions of voter location in a two-party system. Models by Groseclose and others show how the combination of candidate uncertainty about the median voter location, nonzero candidate valence, and policy motivated candidates leads to departures from the median voter prediction. With one policy dimension, either there exists an equilibrium where the candidates do not converge to the median (or any other common position) or there exists no equilibrium. We offer illustrations based on plausible conditions. Under some conditions we show an equilibrium where candidates candidates locate at separate policy positions. Under others, we illustrate the absence of equilibrium.
\end{abstract}




\section{Introduction}

At least since Downs (1957), the median voter's preferred policy has become the presumptive outcome of two-candidate competition with voters choices based on their positions in a one-dimensional policy space. Despite the compelling logic by which, under Downsian assumptions, candidates should converge to the median voter position, they fail to do so. In the United States and the United Kingdom alike, for instance, it is widely agreed that the parties (and their candidates) are even becoming more polarized (McCarty, Poole, and Rosenthal, 2006 and Bruter and Harrison, 2007 respectively). The empirical facts beg the question of why parties and candidates fail to converge as the heralded Downsian model predicts.

It has been obvious to students of electoral politics that the problem must lie with the incompleteness of the Downsian assumptions. Voters choose candidates not simply for the most proximate candidate in issue-space (as the Downs model posits) but also take into account other considerations. Parties and their candidates do not care solely about winning (as the Downs model posits), but also about the policy consequences of elections (Strom, 1990). And parties and candidates do not know exactly voters' positions and their motivations but rather estimate these with uncertainty. Taking these various complications into account, modelers are better able to approximate the game between candidates as they compete for electoral victory (see for instance, Wittman 1983, 1990; Calvert 1985; Londregon and Romer 1993; Aragones and Palfrey 2002; and Groseclose 2001. Adams, Merrill and Grofman 2005, chapter 11 also present an excellent summary of this body of research.) 
The most compelling models incorporate all three features: candidates - with a mix of policy and ideological motivations, "valence issues” motivating voters in addition to policy, and candidate uncertainty about the voters. This uncertainty can take two forms: candidate uncertainty about the valence dimension (Londregon and Romer 1993) or about the median voter's ideal point (Groseclose 2001). With the former, candidates can diverge, with the more popular candidate (advantaged by valence) moving away from the expected median position and the less popular candidate (disadvantaged by valence) toward the expected median position. With the latter, the outcome is reversed. The disadvantaged candidate moves away from the median toward his preferred position while the favorite moves toward the median.

The present paper can be recognized as a variant of the Groseclose model. It proceeds as follows. Section 2 reviews in further detail the general problem of starting with the Downs model but adding assumptions that are realistic, helpful, and tractable. Section 3 introduces the Groseclose model. Section 4 discusses the generality of the conditions under which the Groseclose model applies. Section 5 introduces some illustrations of equilibrium plots, found in the seven figures at the end of this article. Section 6 concludes, followed by a technical appendix further describing our work.

\section{Spatial models of competition and the problem of non-convergence.}

Elections are often modeled as a game between competing parties (or candidates) vying for the allegiance of voters in ideological space. Typically the game involves two candidates fighting over voters whose positions array on a single policy dimension. 
However, voters can have non-policy motivations and candidates can care about policy as well as election. Moreover, policy choices might involve more than one dimension.

The standard starting point of this discussion is the Downs (1957) model of twocandidate competition. The two candidates are solely concerned solely about winning elections. Voters are arrayed on a single left-right policy dimension and vote on the basis of which candidate is closest to their views. The Downs result, which is quite intuitive, is that the candidates will converge toward the center—-toward the median voter. If each candidate is at the median, the election is a tie. This result is also the Nash equilibrium, which means that if either candidate departs from the median position, he or she loses the election.

Despite the intuitive appeal that candidates gain votes by moving to the center, the Downs model obviously is incomplete. Let us describe the ways:

1. Voters vote on the basis of other things besides positions on a dominant policy (or ideological) dimension. The result is that adopting the median voter position is no necessary guarantee of electoral success.

2. Second, like voters, candidates are motivated (at least in part) by policy. Thus they are motivated by policy plus winning and holding office. Their attraction to policy provides them with the incentive to risk diverging from the median voter position.

3. Third, with voters caring about other things besides policy issues, it may be that one candidate holds a net advantage apart from issues. This advantage could be due to vestigial party loyalties or candidate charisma, or something else. The theoretical literature describes this asymmetry of candidate popularity as a 
“valence” advantage. A candidate’s valence advantage means that, in practice, both parties (or candidates) espousing the median position does not result in a tie. It also means that the median voter position may not be the best position for a candidate who starts with a handicap.

4. Fourth, there may be uncertainty in the minds of the actors—so that voters might not know candidate positions and candidates may be unsure of the electorate's positions. Uncertainty by voters about candidates (e.g., Alvarez, 1997) will not concern us here. But uncertainty by candidates about voters will.

5. Fifth, voters can be motivated by policy on more than one dimension, a situation which according to theory can provoke all hell to break loose. With multiple dimensions, Downs's orderly median voter theorem breaks down in favor of unruly indeterminacy and the specter of endless cycling.

All of these considerations add complications and spice to the voting game.

Real-world candidates typically diverge from the median voter position. And they tend to be ideologically fixed, without the instability that non-equilibrium results would imply. Yet, variations on the Downs model typically project the counterfactual persistence of candidate convergence to the median or similar location. And they often lead to at least the possibility of no candidate equilibrium.

Most post-Downsian theoretical treatments of the two-candidate game involve trying to undo the likely lack of equilibrium in multiple dimensions or to convincingly model candidate divergence. As discussed below, the present article deals with both the equilibrium problem and the convergence problem. The ideal is to present a model that provides an equilibrium solution involving candidates who diverge ideologically rather 
than the usual counterfactual prediction of convergence, and the real world implication is to understand the circumstances under which no equilibrium will be found.

\subsection{Probabilistic Voting}

Under what conditions does the candidate game have an equilibrium? That is, under what conditions is there a Nash equilibrium joint location in policy space from which neither candidate has an incentive to depart? The alternative is no equilibrium—no solution to the game and no particular prediction about candidate behavior. With voters voting solely based on issues but with relevant issue preferences lying on more than one dimension, the game generally lacks an equilibrium. Equilibrium in this case can be restored via the realistic assumption that vote choice is a (sufficiently) probabilistic rather than deterministic function of the voter's position in ideological space. The new equilibrium is a weighted mean of voter positions on the various issue dimensions where voters are weighted by their marginality, or the degree to which candidates are uncertain of their vote choice (see, e.g., Enelow and Hinich 1984, Erikson and Romero 1990).

Interestingly, while models of probabilistic voting increase the likelihood of equilibrium in multidimensional models, they do so by taking away the preferred position of a single dimension in terms of imposing order. Just as probabilistic voting greatly expands the likelihood of an equilibrium in multiple dimensions if the voters' error terms are sufficiently large, it also creates the likelihood of non-equilibrium in one-dimensional models if the voters' error terms are sufficiently small (but not zero). With one dimension perfectly determining voter positions (and no valence imbalance), the median voter position triumphs. With a small amount of candidate uncertainty of their vote choices 
(with candidates seeing vote choices as probabilistic function of ideal points) there is no equilibrium. With sufficiently large uncertainty there is an equilibrium at the weighted mean.

With probabilistic voting, while the candidates know individual voter choices only with error, they know the aggregation of the voters' verdict. Thus, with probabilistic voting, candidates converge to a weighted mean that provides their best response to their opponent's best position. But if one candidate enjoys a "valence” advantage due to nonpolicy considerations, that candidate becomes the certain winner. Thus, unsatisfyingly, the losing candidate moves to his best position in terms of vote share, which happens to be his certain loss. Ironically, probabilistic models have too much certainty in that they imply a known valence aspect which, rather than candidate behavior, determines the winner.

\subsection{Candidate Motivation, Uncertainty, and Convergence}

In fact, why don't candidates converge as the simple Downs model predicts? Instead of always competing for the median voter, the US Democratic and Republican parties (and the UK Labour and Conservatives) are polarized with competing left and right positions (of course, to degrees that vary over time and geographical space.). Models subsequent to Downs have made important modifications. Some models allow candidates to pursue their policy preferences as well as their electoral goals, and allow some uncertainty about the outcome. But this by itself does not result in a prediction that fits the data as it can only restate a divergence of candidate positions. Models that include candidate policy goals still leave the puzzle of why candidates don't move toward the 
center where they increase their chances of winning rather than accepting a lottery between two competing ideological candidates.

One might expect a candidate with both policy and electoral goals to edge toward her preferred ideological position. However an important consideration is the adverse policy consequence if an ideologically distasteful opponent is elected. The costs of losing include not only losing office but also suffering under the opposition party’s ideological regime.

As Calvert (1985) has shown, if there exists an equilibrium in the game with uncertainty about the median voter position plus mixed motives by the candidates, it remains in the close vicinity of the median voter's position. And once uncertainty about the median voter's location is introduced, even in one dimension, there is no guarantee of the existence of an equilibrium location. Thus, the added realism of candidate uncertainty plus policy motivated candidates leaves us unsatisfied.

\subsection{Valence}

In the Calvert-type model, no bias exists, so that at equilibrium, each candidate has an equal chance of election. The convenience of this assumption is that maximizing the expected vote margin is identical to maximizing the chance of victory. Suppose we introduce the complication of bias in the form of a valence advantage to one candidate. Now, maximizing victory is different from maximizing the expected vote. The payoff is a set of interesting results.

"Valence" refers to the candidate advantage where one candidate is more popular than the other. Without valence (bias), when the candidates are similar in their policy 
positions or at some other joint equilibrium, the expected outcome is a tie. With non-zero valence (one candidate favored), uncertainty about the location of the median voter, plus candidates who care only about winning, there is an absence of equilibrium even in one dimension.

The intuition is simple. The underdog knows with certainty that he will lose if he copies the advantaged candidate. Consequently the underdog seeks a more extreme position in order to create an ideological opening between him and the advantaged candidate. This allows the possibility, perhaps slim, that the underdog is close enough to the uncertainly located median voter to offset the leading candidate's valence advantage. Meanwhile, no matter where the underdog goes in one-dimensional ideological space, the leader will chase him and copy his position so that with the same ideological positions, the leader's valance advantage ensures the leader's victory. The net result is an endless game with one candidate forever chasing the other. There exists an analogy to yacht racing. As the race's end approaches, the leader does not want to see the lagger take a different and possibly more successful course to the finish line. If they are on the same course, the leader will stay ahead. (For a general discussion of pursuit theory, see Nahin, 2007). In this game with an uncertain median voter location and valence asymmetry plus election-seeking candidates, the only equilibrium that can be achieved is from the unsatisfying artifice of a mixed strategy (Aragones and Palfrey, 2002). ${ }^{1}$

Suppose, however, we add a dose of policy-seeking on the part of the candidates so that they want to win but also care about policy. Now our model includes candidates

\section{Endnotes}

${ }^{1}$ See also Ansolabehere and Snyder, 2000. 
who are both office-seeking and policy-seeking, asymmetrical candidate valence, and candidate uncertainty about the location of the decisive median voter.

A key here is the presence of a decisive median voter who is decisive even though individual voters' decisions presumably are known only probabilistically. This can be accomplished at the sacrifice of assuming a one-dimensional model where the voters to the left of the median voter appear as the symmetrical opposite of the voters on the median voter's right. In this instance, the decisive median voter is also the (weighted) mean voter as well. ${ }^{2}$

\section{The Groseclose Model}

In an important article, Groseclose (2001) has elaborated on the properties of the candidate game with non-zero valence (one candidate favored), uncertainty about the location of the median voter, and candidates who care about both policy and winning. The following is now known. The highlights are:

1. If there exist an equilibrium, the disadvantaged candidate will move away from the center toward his preferred position, while the advantaged candidate will move toward the center from her preferred position.

\footnotetext{
${ }^{2}$ Since we are concerned with valence as it affects the median voter, the source of the valence advantage need not simply be perceptions of candidate competence or charisma, as is usually presented. The valence could be simply party identification, as when one candidate has an advantage due to the district being favorable to the candidate's party in terms of long-standing partisanship.
} 
2. There may not be an equilibrium. Even in one dimension, the game may not have a solution (except possibly involving the artifice of allowing mixed strategies—as if candidates roll dice simultaneously). As the uncertainty about the median voter declines toward zero, the game reverts to the Aragones and Palfrey game where the leader chases the underdog,

The first of these two points is the better understood. Less attractive candidates may have little hope of winning, but their only hope may be that the median voter, whose position is uncertain, may by some slim chance share their position. If the disadvantaged candidate mimics the position of the dominant candidate, the disadvantaged candidate will lose with greater certainty.

The second point is that as uncertainty shrinks the equilibrium is lost. This absence of an equilibrium means that no matter how one candidate responds (in terms of ideological positioning) to the opponent's positioning, the opponent can improve his or her electoral outlook by moving. This would be followed by the first candidate repositioning, and so on. In short, there could be no set of dominant candidate and dominated candidate positions whereby neither candidate has an incentive to move.

Groseclose draws his rich set of conclusions with a stylized model where the candidates' preferred policy positions are equidistant from the median voter and where except for contrasting policy preferences, the candidates’ objective functions and perceptions of the median voter are identical. Although seemingly restrictive, these assumptions are useful simplifications for obtaining comparative statics. ${ }^{3}$

\footnotetext{
${ }^{3}$ For extensions to multiple parties and dimensions, see Schofield (2004).
} 


\section{Generality of the Groseclose Model}

In a two-candidate race, candidates try to maximize not their vote percentage but rather their probability of winning the majority of votes. In the Groseclose model, this goal is reduced to maximizing the probability of winning over one pivotal voter- - the median voter. We should consider the source of this simplification. Groseclose is vague on the details, while Adams et al. (2005) appear to assume that the median voter is decisive because voter decisions are deterministic (rather than probabilistic) functions of candidate proximity and valence. In other words, if valence were zero, voting would be a deterministic function of candidate proximity and therefore the median voter determines the outcome. In a deterministic voter world, valence adds a perception of liking or competency regarding the candidates that is uniform for all voters. That is, all voters share the same relative liking versus disliking for the two candidates independent of the voters' issue differences.

These assumptions of course are not realistic. A realistic model would have voters voting probabilistically so that their decisions are not fully determined by issue positions, valence, and other variables one can measure. Rather, voters have individual error terms to their utility calculus based on considerations the candidates cannot observe. Similarly, it may not be realistic that candidate valence is uniformly perceived by voters. In fact valence perceptions could be non-uniform. They could also vary systematically as a function of voter ideal points.

Fortunately, it is possible to state a very general set of conditions under which the median voter is decisive. In probabilistic models with quadratic loss functions, the decisive voter is not the median voter, but rather the weighted mean voter where the 
weight is determined so that voters whose decisions are more certain carry less weight (Enelow and Hinich 1984, Erikson and Romero 1990). It might be the case, for instance, that voters on one side of the median are more certain in their choice. Thus, for the median to be decisive, the median must also be the appropriately weighted mean voter. This results whenever the density of voter ideal points is symmetric around the median, ${ }^{4}$ as with (but not limited to) a normal distribution). ${ }^{5}$

For the candidate, fixating on the median voter as pivotal can serve as a reasonable approximation for focusing on electoral strategy, even when the symmetry of voter ideal points is only an approximation. The advantage of allowing voting to be probabilistic is that it is realistic. The advantage of allowing variable versions of voters' valence perceptions is that it incorporates other causes of the vote such as party identification. For instance, leftists may dislike the right party candidate more than do right party supporters.

\section{Equilibrium Plots}

Whereas Groseclose illustrates his theoretical results with a stylized example, we consider further variations in the input of candidate characteristics to observe the resultant equilibrium or its absence. We employ a parameterized version of the

\footnotetext{
${ }^{4}$ With symmetry, and noting that $M=$ the median voter's position, $M+\bar{Z}_{i}$ at point $M+\psi$ will be equal to $-\bar{Z}_{i}$ at $M-\psi$.

${ }^{5}$ It must also be assumed that the density of voters' issue weights (relative to their individual error terms) is symmetrical around the mean.
} 
Groseclose model, with quadratic loss as the functional form of candidate and voters' issue loss. Our simulations are performed computationally over grids involving policy choices of two candidates, the Democrat with a preferred policy position to the left of the median voter and the Republican whose preferred policy position is to the median voter's right.

The simulations work as follows. For each set of assumptions, we first set the Democrat's platform at a discrete set of positions, separated by small regular intervals (from -2 to 2 in 0.05 increments). For each possible platform position, we determine the Republican candidate's best response. Then we repeat the procedure, estimating the Democrat's best responses to the Republican's possible positions. If a fixed point exists (i.e., where the candidates' positions of best responses equal their opponents') then the corresponding platforms represent an equilibrium. If the best responses are graphed on a common set of axes, the best response curves will cross at this point. When the lines fail to cross, there is no equilibrium. Our full procedure of simulations is detailed in the appendix.

For most of the illustrations that follow, the Democrat's preferred position is at - 1 while the Republican's is at +1 . The median voter's position always has an expectation of zero. Our starting point regarding the variance of the median voter position is that the variance is 0.01 (a standard deviation of 0.10 ). With this value for the standard deviation, candidates see it as extremely unlikely that the median voter actually approaches the candidate’s own preferred position.

First consider Figure 1. Here, neither candidate has a valence advantage (which is expressed by the mention $\operatorname{pr}($ Dem win $)=50 \%$, so that the Democratic candidate is 
expected to have a $50 \%$ chance of winning the election). Each candidate cares deeply about policy. In fact, given the assigned desire to win of 0.25 (relative to 1.0 for policy), each candidate would trade victory at the median position if the opponent's victory means moving policy roughly one tenth the distance from the median to the candidate's preferred position.

(Figure 1 about here)

We see an equilibrium outcome near zero in the northwest quadrant of the graph where the two curves cross. The figure gives the exact coordinates of the equilibrium point, at 0.6, 0.6. The solid curve represents the Republicans' best response (on the y axis) given the various possible Democratic positions on the x axis. The dashed curve represents the Democrats' best response (on the $\mathrm{x}$ axis) given the various possible Republican positions on the y axis. In each case the best response incorporates both the electoral and the policy motivations. At the crossing-point, neither candidate has an incentive to change her position given the opponent's position; thus there exists a Nash equilibrium. Note that even with a dominant interest in policy outcomes, the candidate equilibrium is close to a candidate convergence, with the Democrat slightly left of the expected median position and the Republican slightly to the right (up on the graph).

Figure 2 shows the result with the combined circumstances of less policy concern by the candidates but also an imbalance on valence. Here, the candidates' desire to win is 1.0, so that now each candidate would trade victory while positioning at the median voter position for a loss if the winning opponent positions herself at our candidate's preferred position. We also add a mild valence advantage to the Democrat of 0.1 . This means that in expectation, the indecisive voters who are equally attracted to the two candidates 
would be at about 0.25 , or slightly right of center. (Their slight valence advantage for the Democrat is equivalent to their slight policy advantage for the Republican.).

(Figure 2 about here)

In Figure 2, the equilibrium outcome is in the northwest quadrant, but farther from the voter median. The Democrat can move this far left because of the cushion of her valence advantage. The Republican is farther right (up) than in Figure 1, with no incentive to move closer to the center because doing so gains little further chance of winning and loses policy utility. Note that with the Democrat located in the vicinity of the expected median voter position, the Republican candidate would become indifferent to moving left for a greater likelihood of victory than right for greater policy satisfaction. The valenceadvantaged candidate - the Democrat—is the closer to the median voter.

Figure 3 makes only one change to the assumptions of Figure 2 but it is an important one. It increases the desire to win to an arbitrarily high number, meaning that each candidate now only cares about winning. The result is now the absence of an equilibrium, as the two curves do not intersect. (Note the discontinuity in the Republican's curve in the vicinity of the expected median.) The disadvantaged Republican tries to differentiate herself by separating her position from the Democrats' while the Democrat chases the Republican (in the spirit of Aragones and Palfrey 2001).

(Figure 3 about here)

Figure 4 is also a mild departure from Figure 2 in terms of the assumptions. Here, the Democrat's valence advantage doubles to 0.2. The equilibrium coordinates move even farther left from the median (compared to Figure 2) as the Democrat sees little electoral risk in moving left. Meanwhile, the Republican moves right (up), compelled to 
differentiate on policy in the face of the major valence disadvantage. Here, the likely loser, the Republican is actually closer to the expected median. Although closer to the center, the Republican's slim issue advantage cannot overcome the larger Democratic valence advantage.

(Figure 4 about here)

Figure 5 returns to the assumptions of Figure 1, except now adding greater uncertainty in the form of a 0.3 standard deviation so that the median voter is now estimated to be at about 0.0 but with a confidence interval of about plus or minus 6 . Here the heavily policy-motivated candidates move farther toward their preferred positions than in Figure 1. Note that although each candidate's curve shows a discontinuity, they do manage to cross at an equilibrium point.

(Figure 5 about here)

Figure 6 offers for illustration an equilibrium analysis when there is asymmetry in the candidates' motivations and perceptions, as well as valence. Here, the Republican is far more interested in winning compared to policy but has greater uncertainty about the median voter's location and is disadvantaged on valence. We see a nonconvergence but close to the center, with the advantaged Democrat farther from the expected median than the Republican. The idealistic Democrat cares much more about policy than winning, and sacrifices the probability of winning for a more leftist policy should she win.

(Figure 6 about here)

Finally, Figure 7 offers still one more illustration where for the first time we create asymmetry in the candidate preferred positions relative to the expected median. 
Here we give the Republican a very slight valence advantage, greater interest in winning, less uncertainty about the median’s location plus a more moderate position (0.6) relative to the Democrat (1.0 as usual). Here the Republican stays close to the median as long as the Democrat is slightly left of the expected median. The Democrat's best response is slightly left of the expected median in order to differentiate.

(Figure 7 about here)

Hopefully these seven illustrations serve to advance the intuition for the Groseclose model and to hint at how the results shift as we vary the moving parts. The graphs show how under reasonable but varying conditions, we get the result that in equilibrium two candidates do diverge - in varying amounts_-in their policy positions. At the same time (see Figure 3), the outcome may be a non-equilibrium-even in one dimension-if the policy motivation is weak and the valence is strong.

\section{Conclusions}

We close with an agenda for further work. Although we have strived to offer variations in the Groseclose model that depict real-world conditions, the replication of electoral reality—or alternative electoral scenarios could be expanded if not improved. For instance, if we accept the technical condition of radial symmetry, we could model voters and candidates in two-dimensional issue space. Or, we could abandon all assumptions of voter symmetry in issue space with its convenience of modeling one median voter. Instead, we could model all sizes and shapes of voter preferences, in one or more dimensions, with skewed distributions and asymmetrical weighting of issues-plus the requisite uncertainty, policy motivation, and valence. To do so would require intensive 
computation-integrating over a density of voters rather than the median voter alone. It is worth considering, however, how the progressive further relaxation of the assumptions that have led us to expect systematic convergence between parties or candidates in two party systems is necessary for us to better understand the paradoxical candidates' positionings that we witness in real life.

\section{Acknowledgements}

This research was supported by an LSE-Columbia Seed Grant. An earlier version of this paper was presented at the Conference on the Dynamics of Partisan Position Taking, Binghamton University, March 22-23, 2007. We thank John F. Adams, Charles Cameron, Michael D. McDonald, Michael Peress, Kelly Rader, Norman Schofield, and an anonymous referee for their helpful comments

\section{Appendix}

In this technical appendix, we first describe a parameterized version of the Groseclose model, with quadratic loss as the functional form of candidate and voters' issue loss. Second, we describe how we transport the model to graph the equilibrium location under varying scenarios.

\section{Legend:}

$U=$ Utility of the Democratic Candidate

$Z=$ Utility of the median voter

$B=$ bliss point (ideal point) of the Democratic candidate

$X=$ the Democratic candidate's public policy position, the object of our attention

$R=$ the Republican candidate's public policy position

$0=$ zero=expectation of the median voter position

$P=$ probability of a Democratic victory 
$1=$ one=Democratic candidate's policy weight

$W=$ value of winning to Dem. candidate (“desire to win,” as relative to policy weight)

$u=$ error in estimate of the median voter position

$s=$ standard deviation of the median voter position $(M)$

$M=0+u=$ median voter position where standard deviation of $u=s$

$a=$ valence or intercept term for the median voter, the median voters' relative liking for the Democrat over the Republican.

The Median voter's relative utility for the Democrat minus that for the Republican:

$$
Z=-(X-M)^{2}-(R-M)^{2}+a
$$

The median voter is decisive. We model the decision of the Democratic candidate

$U=-(B-X)^{2} P-(B-R)^{2}(1-P)+W P$

$U=P\left[R^{2}-X^{2}-2 B(R-X)+W\right]-(B-R)^{2}$

$P=\frac{1}{\sigma \sqrt{2 \pi}} \int_{-\infty}^{Z} \exp -\frac{u^{2}}{2 \sigma^{2}} d u$

where

$$
Z=a+R^{2}-X^{2}
$$

and

$$
\sigma=2 s(R-X)
$$

Elaborating,

$$
U=\frac{1}{2 s(R-X) \sqrt{2 \pi}} \int_{-\infty}^{a+R^{2}-X^{2}} \exp -\left\{\frac{u^{2}}{2 s^{2}(R-X)^{2}}\right\} d u\left[R^{2}-X^{2}-2 B(R-X)+W\right]-(B-R)^{2}
$$

Rearranging,

$$
U=\frac{R+X-2 B+W\left\{\frac{1}{R-X}\right\}}{2 s \sqrt{2 \pi}} \int_{-\infty}^{a+R^{2}-X^{2}} \exp -\left\{\frac{u^{2}}{2 s^{2}(R-X)^{2}}\right\}-(B-R)^{2}
$$

Differentiating,

$$
\frac{d U}{d X}=\frac{W+(R-X)^{2}}{2 s \sqrt{2 \pi}(R-X)^{2}} \int_{-\infty}^{a+R^{2}-X^{2}} \exp -\left\{\frac{u^{2}}{2 s^{2}(R-X)^{2}}\right\} d u+\frac{W+(R-X)^{2}}{4 s^{2} \pi(R-X)} \exp -\frac{\left(a+R^{2}-X^{2}\right)^{2}}{2 s^{2}(R-X)^{2}}
$$


where

$$
\frac{d U}{d P}=Y^{2}-X^{2}-2 D(Y-X)+W
$$

and

$$
\frac{d P}{d X}=\frac{1}{2 s(R-X) \sqrt{2 \pi}} \exp -\frac{\left(a+R^{2}-X^{2}\right)^{2}}{2 s^{2}(R-X)^{2}}
$$

The next step is setting $U$ to zero to find its maximum as a best response to $R$. Then we do the mirror-image modeling for the Republican candidate, finding the Republican’s best response (now labeled $Y$ ) to the Democratic position $(X)$. We ask under what assumptions present a combination $(X, Y)$ where $X$ and $Y$ are the best responses to each other, and we seek its location, given the assumptions of the model.

Following is how we find equilibria and plot the illustrative graphs.

The necessary inputs are: Democratic candidate ideal point, Republican candidate ideal point, Democratic candidate desire to win, Republican candidate desire to win, uncertainty about the median voter, and valence advantage for the Democratic candidate. First, an arbitrary position from the Republican candidate is chosen (0.3). Next, the Democratic best response is determined via applying the model and R's built in optimization function. The optimization function is similar to Newton-Raphson; here's what $\mathbf{R}$ says about it:

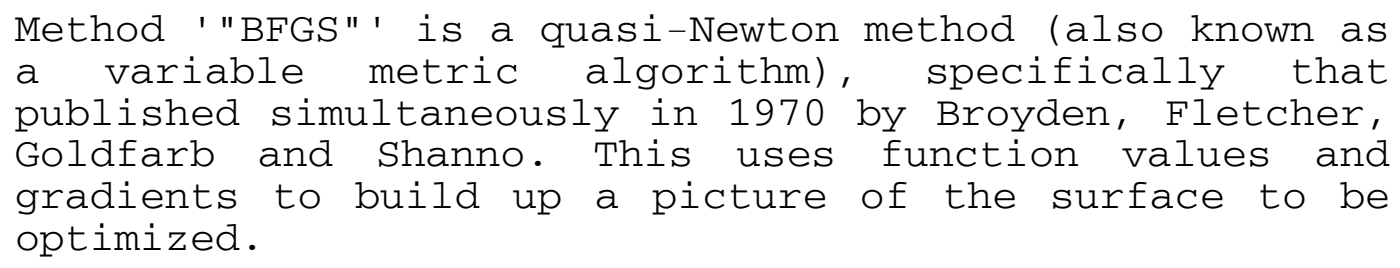


This "best response" position is assigned to the Democratic candidate. The "best response” for the Republican candidate (given this Democratic position) is then determined in the analogous way. This iterative process is repeated 100 times or until both candidate positions do not change by more than $10^{\wedge}-4$. If this convergence condition is not met after 100 iterations, we assume there is no equilibrium. More formally, we assume that the equilibrium is an attractive fixed point. If this assumption is violated, we may make errors of omission. The above algorithm has found all the equilibrium presented in the figures in this paper.

For the plots themselves, the "best response" procedure is applied to both candidates, letting their opponent's position vary from -2 to 2 at intervals of 0.05 . The Democrat's best response at each of the Republican's positions is plotted with a blue dashed line connecting each of these best response points. The Republican's best response is plotted using a solid red line.

In the lower right, the equilibrium positions of the Democratic and Republican candidates are reported (if an equilibrium exists). The Democratic probability of winning at equilibrium (the Democrat's uncertainty—if it differs from the Republican's—taken as given) is also reported. 


\section{References}

Adams, J. F., Merrill, S., and Grofman, B. (2005). A unified theory of party competition. New York: Cambridge University Press.

Alvarez, M. (1997). Information and elections. Ann Arbor: University of Michigan Press.

Ansolabehere, S. D.and Snyder, J. M. (2000). Valence politics and equilibrium in spatial election models. Public Choice. 03: 327-336.

Aragones, E. and Palfrey, T. R. (2002). Mixed equilibrium in a Downsian model with a favored candidate. Journal of Economic Theory 103: 131-161.

Bruter, M. and Harrison, S. (2007). United Kingdom. In Deloye, Y. and Bruter,M. (eds) Encyclopaedia of European elections. Basingstoke: Palgrave Macmillan.

Calvert, R. L. (1985). Robustness of the multidimensional voting model: Candidate motivations, uncertainty, and convergence. American Journal of Political Science 29:69-95.

Downs, A. (1957). An economic theory of democracy. New York: Harper \& Row

Enelow, J. and Hinich. M. (1984). The spatial theory of voting. New York: Cambridge University Press.

Erikson, R. S. and Romero, D.R. (1990). Candidate equilibrium and the behavioral model of the vote. American Political Science Review 84 (December): 1103-1125.

Groseclose, T. (2001). A model of candidate location When one candidate has a valence advantage. American Journal of Political Science. 45 (October): 862-886.

Londregon, J. and Romer, T. (1993). Polarization, incumbency, and the personal vote. In 
Political economy: Institutions, competition, and representation. Barnett, W.A., Hinich, M., and Schofield, N. New York: Cambridge University Press.

McCarty, N., Poole, K., and Rosenthal, H. (2006). Polarized America. Cambridge: MIT Press.

Nahin, P. J. (2007). Chases and escapes: The mathematics of pursuit and evasion Princeton: Princeton University Press.

Schofield, N. (2004). Equilibrium in the spatial valence model of politics. Journal of theoretical politics. 16: 447-481.

Strom, K. (1990). A behavioral theory of competitive political parties. American Journal of Political Science. 34.2: 565-598.

Wittman, D. (1983). Candidate motivation: A synthesis of alternatives. American Political Science Review. 77: 142-57.

Wittman, D. (1990). Spatial strategies when candidates have policy preferences. In Advances in the Spatial Theory of Voting, Hinich, M. and Enelow, J. Cambridge: Cambridge University Press. 


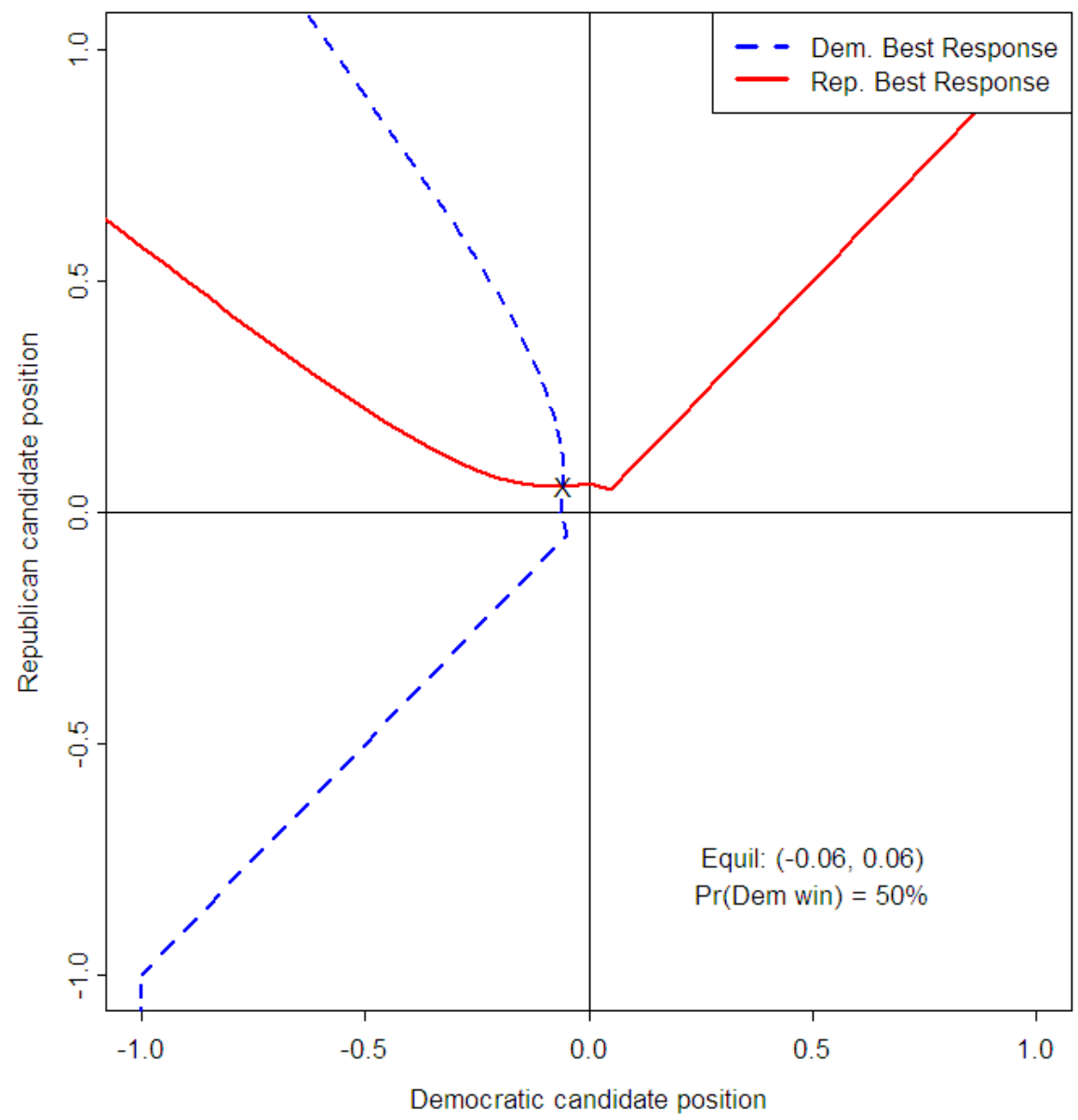

Figure 1. Candidates care mostly about policy; No valence advantage.

AssumPTIONS: Ideal points are -1 for the Democrat and +1 for the Republican; The perceived ideal point of the median voter: mean $=0$, standard deviation $=0.1$. Each candidate weighs policy (quadratic loss) four times the value of winning. Neither candidate holds a valence advantage.

RESULT: The equilibrium is symmetric, with the candidates positioning themselves slightly to the left and right of the median voter for the Democrat and Republican respectively. The two candidates have an equal chance of winning. 


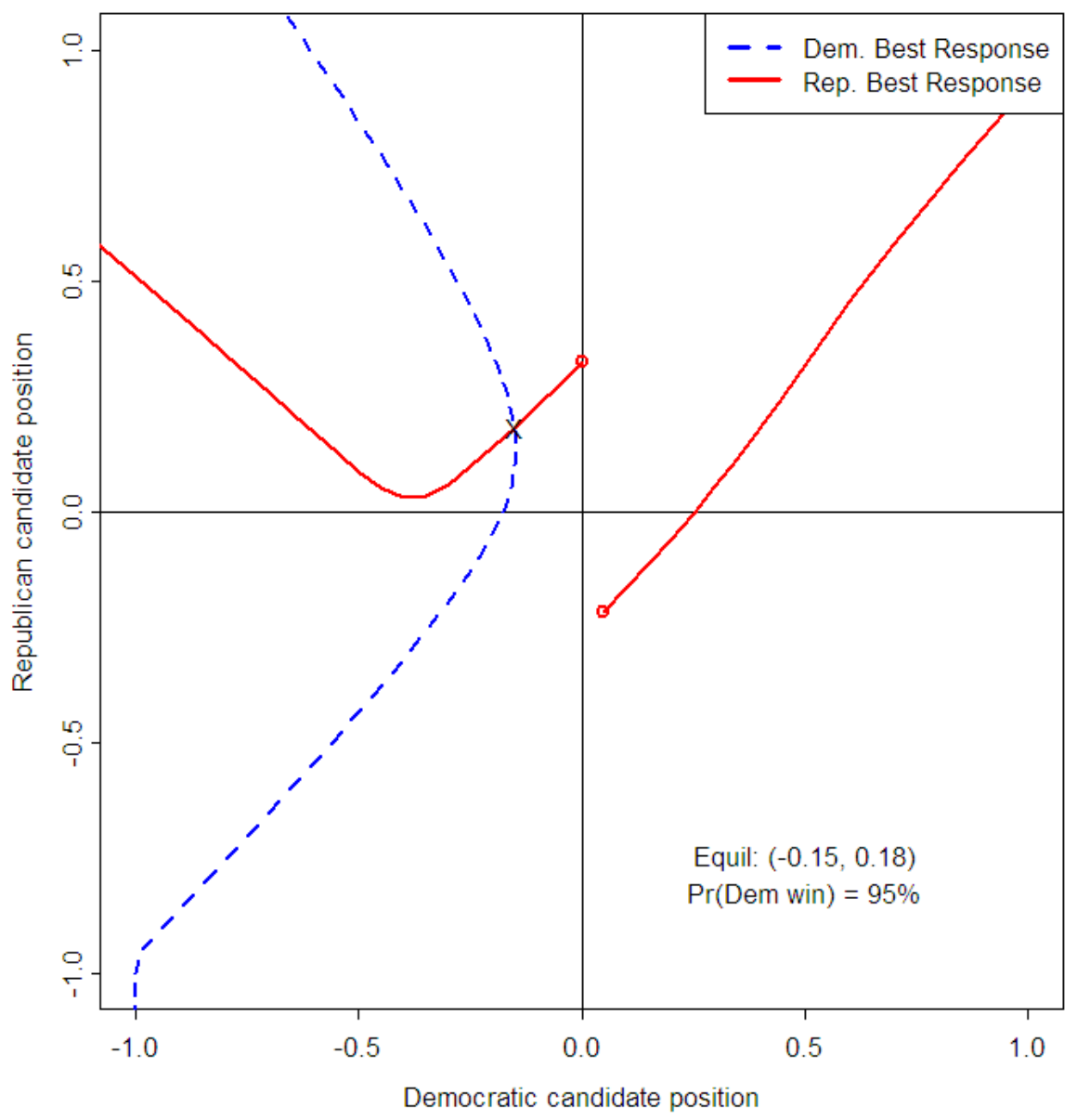

Figure 2. Candidates care about winning and policy; Democrat has valence advantage Assumptions: Ideal points are -1 for the Democrat and +1 for the Republican; The perceived ideal point of the median voter: mean $=0$, standard deviation $=0.1$. Each candidate weighs policy (quadratic loss) equal to the value of winning. The Democrat has a valence advantage of 0.1 .

RESULT: In equilibrium, the Democrat is slightly more centrist than the Republican, which ensures Democratic victory 95\% of the time. The Republican is more extreme than to the Democrat to maximize the utility of the few victories it can expect. 


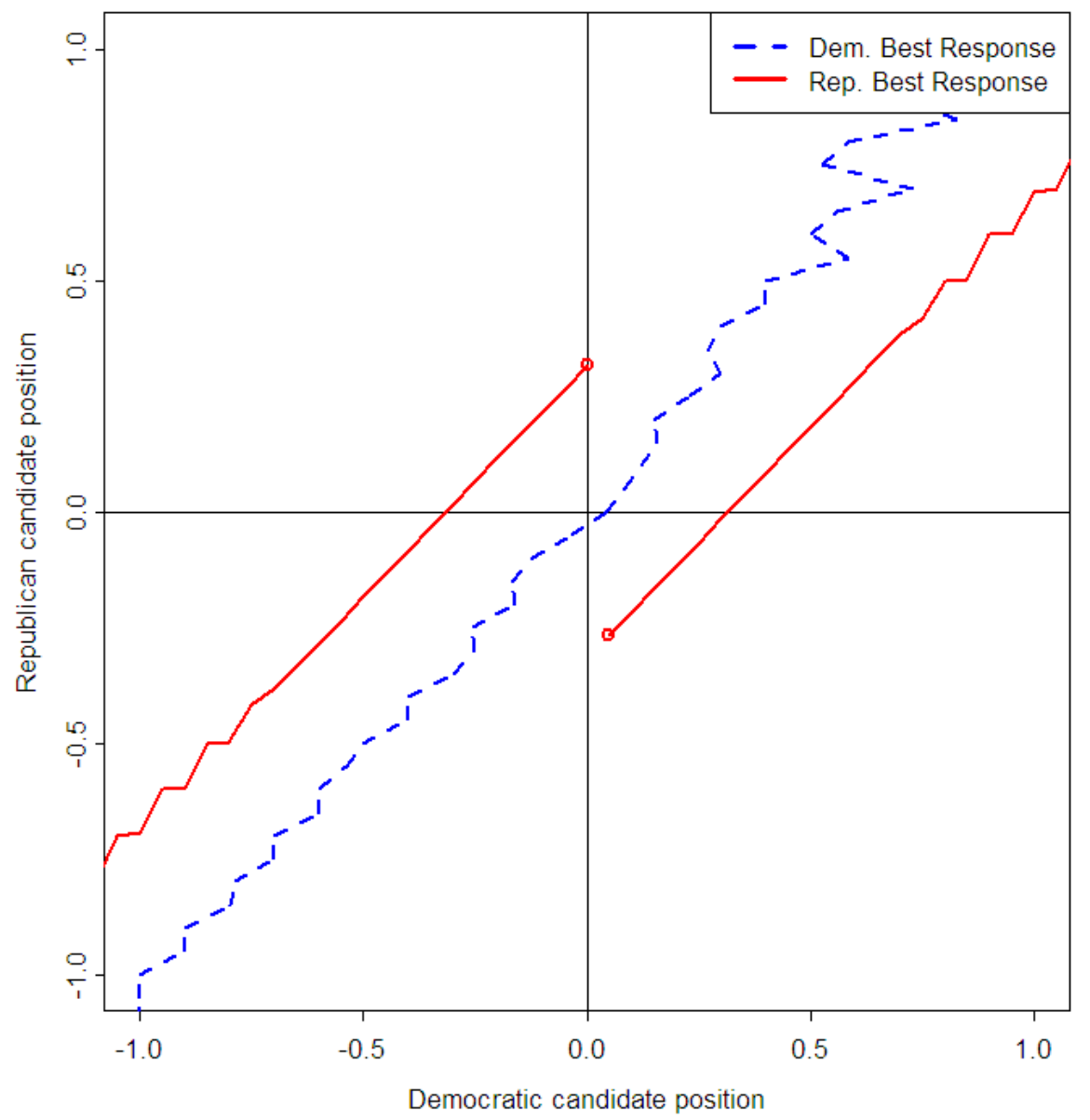

Figure 3. Candidates only care about winning; Democrat has valence advantage. Assumptions: Ideal points are -1 for the Democrat and +1 for the Republican; The perceived ideal point of the median voter: mean $=0$, standard deviation $=0.1$. Each candidate cares only about winning. The Democratic candidate holds a valence advantage of 0.1 .

RESULT: There is no equilibrium, as the Democrat's best response is to eliminate any platform differences by adopting the Republican's platform and the Republican's best response is to create policy separation between the candidates. Candidate ideal points are irrelevant in this simulation. 


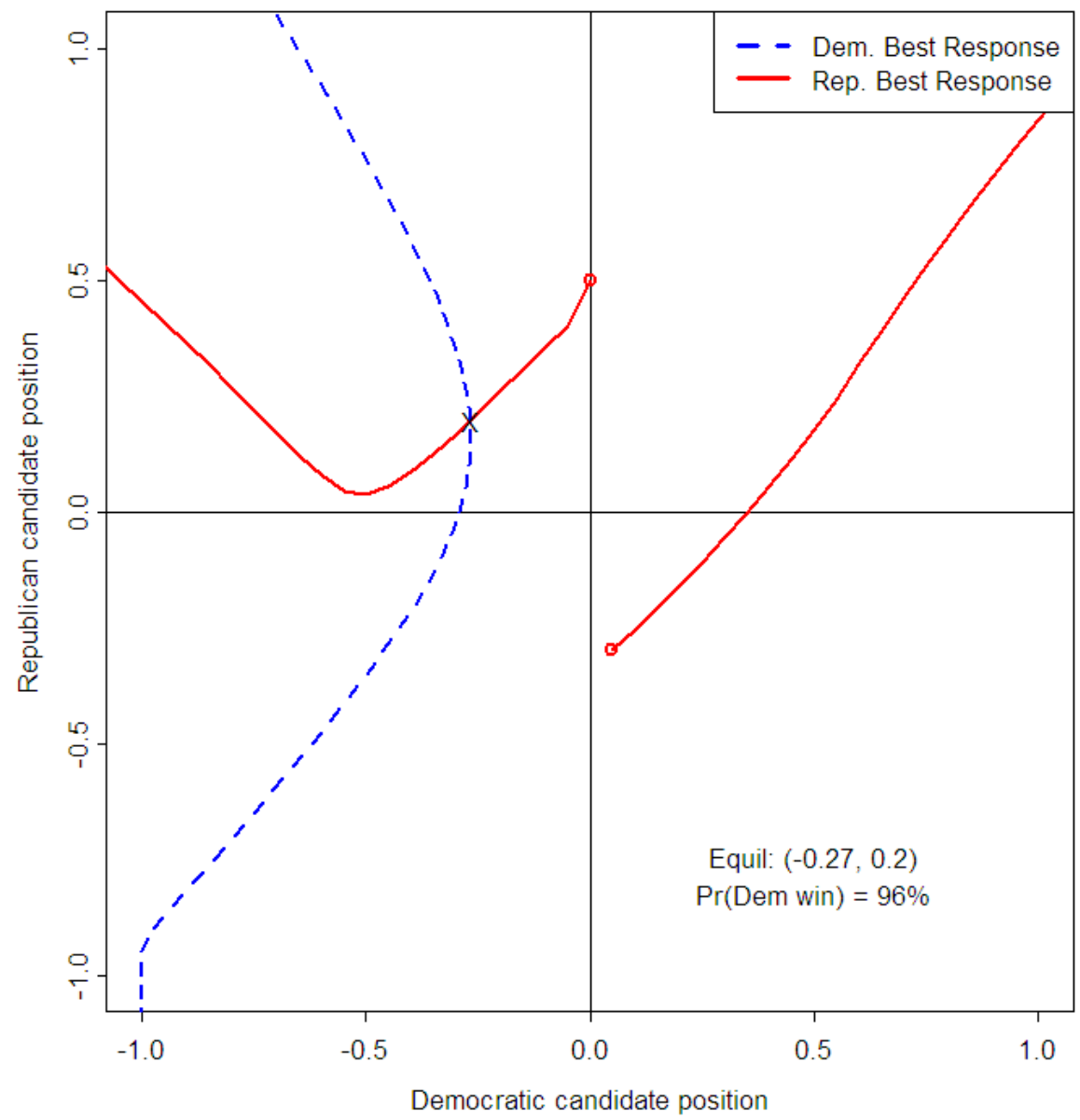

Figure 4. Candidates care winning and policy; Democrat has large valence advantage. Assumptions: Ideal points are -1 for the Democrat and +1 for the Republican; The perceived ideal point of the median voter: mean $=0$, standard deviation $=0.1$. Each candidate weighs policy (quadratic loss) equal to the value of winning. The Democrat has a valence advantage of 0.2 .

RESULT: In equilibrium, the Democrat is slightly more centrist than the Republican, which ensures Democratic victory 96\% of the time. In equilibrium, the Republican is more extreme than the valence-advantaged Democrat to maximize the utility of the few victories it can expect. 


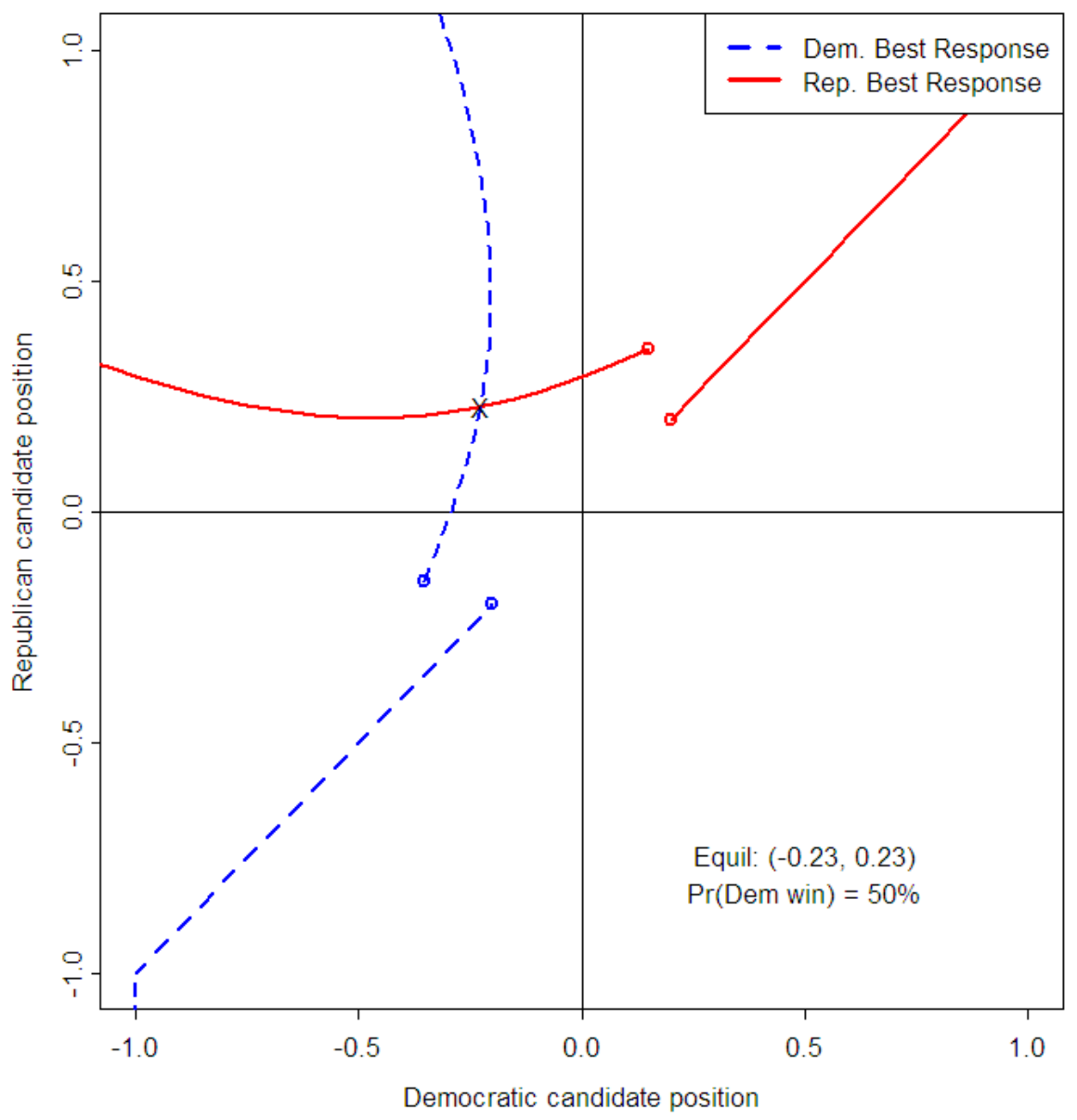

Figure 5. Candidates mostly about policy; High uncertainty about the median voter's location. AssumPTIONS: Ideal points are -1 for the Democrat and +1 for the Republican; The perceived ideal point of the median voter: mean $=0$, standard deviation $=0$. 3. Each candidate weighs policy (quadratic loss) four times the value of winning. Neither candidate holds a valence advantage.

RESULT: The equilibrium is symmetric, with the candidates positioning themselves significantly to the left and right of the median voter for the Democrat and Republican respectively. Each candidate has an equal chance of winning 


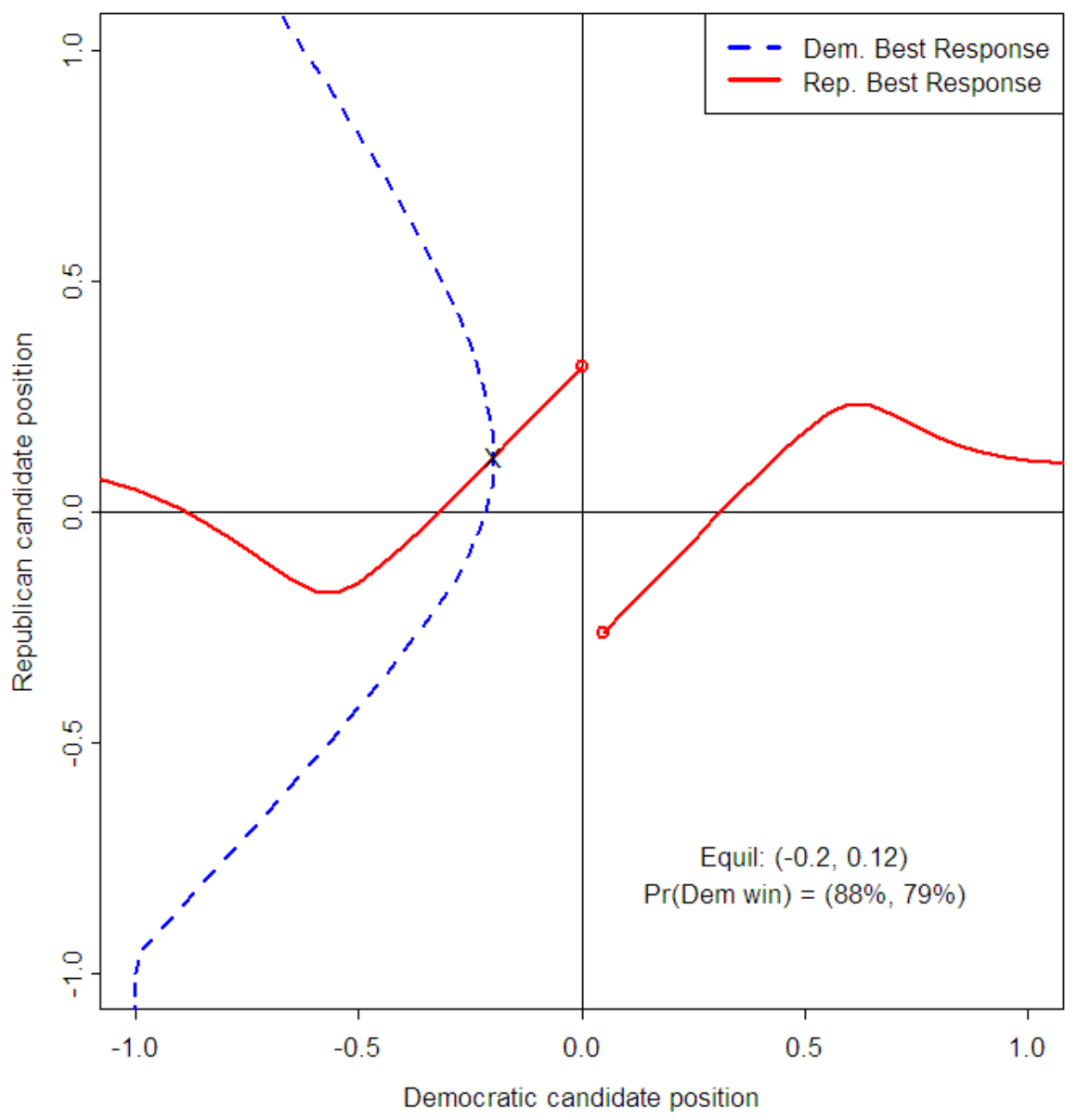

Figure 6. A mixed bag of assumptions. AsSUMPTIONS: Ideal points are -1 for the Democrat and +1 for the Republican. The Democrat's perceived ideal point of the median voter: mean $=0$, standard deviation $=0.1$. The republican's perceived ideal point of the median voter; mean=0, standard deviation=0.15. The Democrat weighs policy (quadratic loss) nine times the value of winning. The Republican weighs winning four times the value of policy (quadratic loss). The Democrat has a valence advantage of 0.1. RESULT: This model simulates a popular policy-oriented Democratic incumbent versus an election-seeking Republican challenger. With a mild valence advantage, the Democrat diverges from the median voter more than the Republican. . Given the difference in uncertainty, the Democrat perceives his/her probability of winning to be $88 \%$, whereas the Republican believes that quantity to be $79 \%$. 


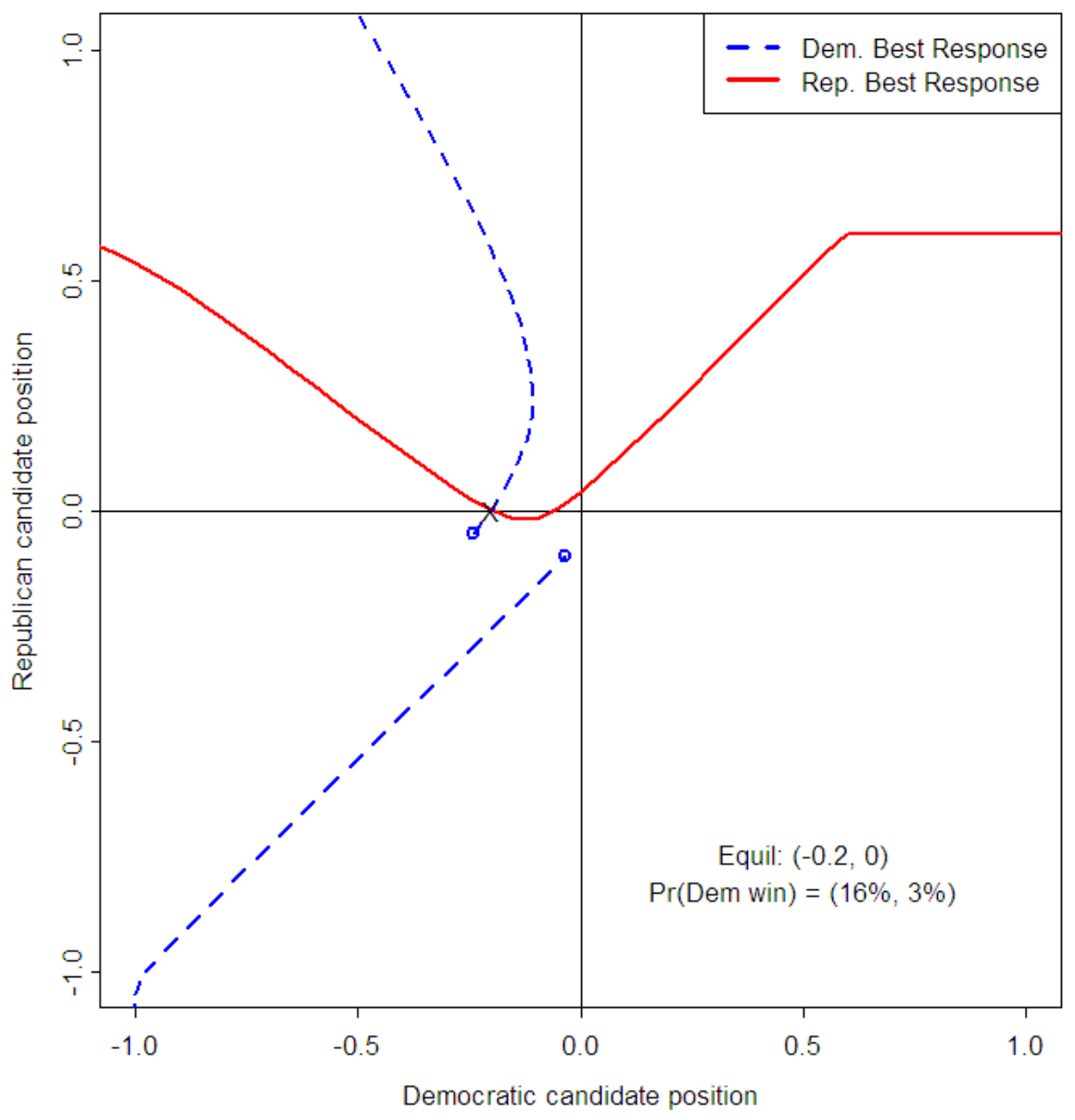

Figure 7. Simulation of a moderate Republican vs. liberal Democrat. AsSUMPTIONS:

Ideal points are -1 for the Democrat and +0.6 for the Republican. The Democrat's perceived ideal point of the median voter: mean $=0$, standard deviation $=0.15$. The republican's perceived ideal point of the median voter; mean $=0$, standard deviation=0.08. The Democrat weighs policy (quadratic loss) four times the value of winning. The Republican weighs winning at $150 \%$ the value of policy (quadratic loss). The Republican has a valence advantage of 0.02 .

RESULT: This model simulates a somewhat popular moderate Republican incumbent facing a more policy-oriented Democratic challenger. The Republican's concern for winning and her accurate knowledge of the electorate push the Republican toward the median voter more than her small valence advantage allows her to move away from the center. The Democrat's idealism and uncertainty result in that candidate positioning himself to the left of the median voter. 